\title{
Possible Utilization of the Artificial Intelligence Elements in the Creation of Remote Experiments
}

\author{
http://dx.doi.org/10.3991/ijoe.v10i1.3110 \\ M. Krbeček ${ }^{1}$, F.Schauer ${ }^{1,2}$ and I. Zelinka ${ }^{1}$ \\ ${ }^{1}$ Tomas Bata University in Zlín, Zlín, Czech Republic \\ ${ }^{2}$ University of Trnava, Trnava, Slovak Republic
}

\begin{abstract}
Remote laboratories start to be an important tool both in science and education. The massive spread of those modern technologies is hampered by the absence of the standardized solutions and difficulties with their implementation by the wide segment of scientists and educators. This paper intends to contribute to the idea how the artificial intelligence may contribute to the remote laboratories controlling programme creation and thus remove the ensuing barrier. The paper describes first the basic scheme of a remote experiment on a typical solution example based on the Internet School Experimental System (ISES) hardware and the state of the art in the compiling of the control programme software. Then it focuses on the basic principles of the artificial intelligence and suggests its use in the developing of remote experiments. This part can serve as an inspiring source of ideas for the future developments in the area, particularly in the consideration about the future of remote experiments. In conclusions are summarized the main contributions of the artificial intelligence, which will affect the development of software for the design of control logic of remote experiments, especially based on the system ISES.
\end{abstract}

Index Terms-Remote experiments, remote laboratories, artificial intelligence, control logic of remote experiments.

\section{REMOTE LABORATORIES - STATE OF THE ART}

The contemporary society is characterized by sharing of resources and assets through the Internet and growing virtualization in ICT. This approach saves the cost of expensive shared devices, available through the network. This trend can be found in a wide range of sectors of human activities in general and in science and teaching processes in particular. Teaching of natural sciences is no exception. Next we intend to describe the situation typical for universities; in other fields the situation is similar. A great deal of attention worldwide at universities and teaching institutions has been devoted to e-laboratories offering access to various real world remote experiments (REs) [15]. The ultimate goal in the creation of teaching support for a teacher are global grids of remote laboratories and their integration into a cloud-system with an easy data retrieving, processing and storing.

Such experiments are mainly designed under the auspices of the universities for the purpose of education (teaching and learning). For this reason usage of some of them is only for students of the particular university in question and access is secured by the user name and password (especially at American universities). In Europe and Australia several projects exist supporting building of open remote laboratories - with free access, either with or with-

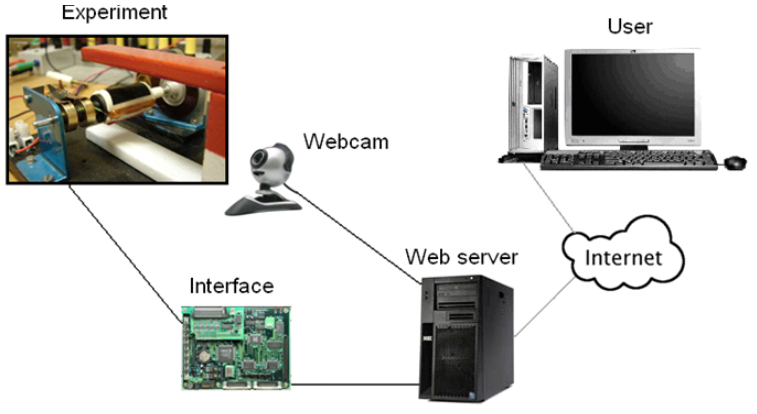

Figure 1. Schematical arrangement of the remote experiment [6]

out registration. Some labs offer to insert client's initials (name, country, e-mail) for voluntary statistical purposes. With necessary exceptions (maintenance, modification, technical issues...) experiments are run on 24/7 scheme [7$10]$.

There are, rather unfortunately, plentiful and not compatible systems for creating computer oriented physical experimental laboratories. But only a part of these systems allows remotely access and control of the devices via the Internet or internal network. From this group, we can select only certain systems that are suitable for the creation of remote experiments for educational purposes $[10$, $11,12]$. The utilization of the other systems is primarily in the areas of development or research. Almost all systems from the group applied for teaching are focused on complex technical tasks and their applications are mainly in academic environment. From this already quite small group we selected a representative which is suitable not only for the university level, but due to its plug and play design it is also suitable for lower levels of the educational process. This unique system is called Internet School Experimental System (ISES) [10].

\section{A. Internet School Experimental System - ISES}

ISES is a powerful tool for process and experiments control, acquisition, collecting and data processing in real time. Let us mention the basic features of the ISES system, more detailed description may be found elsewhere [10]. The basis of the system is ISES board, which is available in several versions, differing depending on the number of inputs/outputs and also on type of communication with the control PC (by PCI card, USB connector, Wi-Fi). To this board are, by a unique connector, plugged in sensors like: ammeter, voltmeter, thermometer, position sensor, ohmmeter, load cell, anemometer, microphones, sonar, light gate, $\mathrm{pH}$ meter, conductivity meter, heart rate monitor, etc. [10]. 
PAPER

Due to its maximum signal transfer frequency (100 $\mathrm{kHz}$ ) the system allows the study of sounds or other dynamic signals. The system allows simultaneous measurement, processing and displaying data via maximum eight input channels, as well as process control via two analogue and two binary output channels. But the uniqueness of this system is its possibility of using the same equipment both for experiments in the laboratory (so-called hands on experiments) and also for their remote versions remote experiments (RE).

\section{B. Remote experiments - not so simple}

Creation of the RE based on the ISES was in the past a relatively complex matter. Though the system itself was perfectly ready for this option due to its plug and play features. A problem arose on compiling of control logic of the remote experiment based on its flow chart. For example the logical sequence (e.g. for reaching the desired temperature) that performs the specified action (relay turn on/off the heat). This controlling logic also has to secure the interactions between the remote users (clients) and the controlling of the experiments (when a client presses the virtual switch on his/her screen then the same action must follow, as if it were the actual power supply button of the device). Until recently all these functions have been addressed by the control file (so called ".psc" file), containing instructions and logic which replaced the "thinking" of the remote experiment and enabled remote control. Compiling of this directly executable file was done by a pseudo programming language whose complexity was far beyond the ability of the layman in the field. Until recently this was a huge obstacle for the spreading of self made remote experiments at all levels of education.

The situation calls for remedy and we intend to help with this paper to contribute with some ideas in the direction of spreading remote experiments among the users with basic knowledge of informatics. Because the RE needs the control logic and there was not a possibility of its complete removal, we choose an intermediate solution. The final product was essentially a graphical and iconic development environment creating control file from a flowchart by using of the pre-prepared control blocks which generated a control file by means of simple questions and choices of the RE designer. This solution enabled to design even very complex and sophisticated RE and will be described in more detail elsewhere [13].

Now the question arises: could we go in resolving of this problem of simplification even further? To create something universal that would simplify this process to few clicks? Or even just a few words spelled by a user? In principle it is feasible by using up-to-date knowledge of artificial intelligence elements and we will try to point out those possibilities in the text below.

\section{INTELLIGENT, EVOLUTION, AND EXPERT SYSTEMS - ALL THIS AND MORE}

As indicated above we will try in the following chapters to concentrate on some intelligent methods of design and creation of remote experiments of future. Under this concept, we can imagine a great many things and possibilities. Let us specify the term of "intelligent methods" as follows: By using these up to now not specified methods, we intend to transfer a part (the size of this part may be different for each solution) of human's thinking and decisionmaking process to a computer. This will make end-users' work easier (on the contrary, the work of developers of this solution will be immensely more complicated).

Everyone surely heard the term "Artificial Intelligence" (AI) and certainly saw many sci-fi movies where the AI enslaved the world, or at least tried it. We disappoint those who began to pay attention on reading the previous sentence; there will not be anything like that in our case. This field is without doubt a major contemporary trend with continuous progress. Today we can see its use in many branches of industry as computer science, finance, health care, heavy industry, transportation, telecommunication, toys and games, aviation and much more. So, why not to attempt to use AI elements also in the creation of RE?

\section{A. Evolutionary algorithms}

In order to better explain systems using AI, let us first explain, what the word "evolution" means. As the best example we can mention theory of Charles Darwin evolution, which describes the gradual evolutionary development of species on the Earth. [14] It is actually a spontaneous process during the course of which life gradually develops and diversifies. Successive crossbreeding is resulting in creation of new organisms and animal species that bear the characteristics of their ancestors. This genetic crossbreeding can have both positive and negative impact on the future organism. By crossbreeding may thus arise even an animal with worse properties for the environment than his ancestors. But here comes the so-called natural selection, which deals this deficiency by its own way and the survival of such kind of animal usually does not take long. We ourselves are the evidence that the evolution really works (it's our skills, our adaptation to the environment and also our developed ability of thinking).

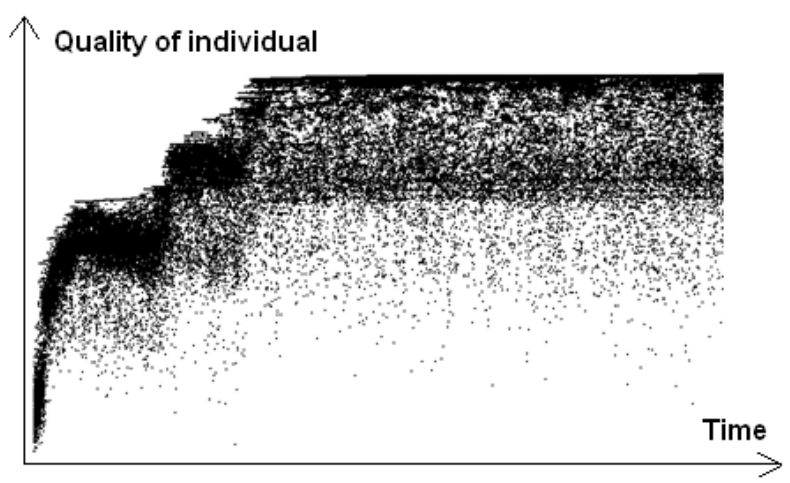

Figure 2. Quality of individuals in the virtual evolution

On the same principle operates the evolution in virtual (computer) environment. Here we use so-called evolutionary algorithms which by successive crossbreeding of certain objects with different properties create their offspring. The first individuals for the start of development can be randomly generated or chosen those who were successful in solving of the problem in the past. The descendants of these individuals share the properties of their ancestors and thus more new objects with new properties are created. As in the nature some created objects do not contribute to the required solution of problems. But the testing of them proves their unsuitability and therefore they are excluded from further evolution (here appears next link to the biological evolution and natural selection). The remaining individuals continue in the evolution until the required quality of the solution is reached. The course of such evolution in computing environments is shown at 
Figure 2. Each point represents one of the offspring. The graph shows the boundary of the quality, which the new offspring cannot cross. Absolutely obvious utilisation of these algorithms is optimization problems, where we are looking for the best solution to a problem [15]. Generally speaking, this method is suitable for systems where we know the desired output but do not know the way to achieve him. Algorithms have been used for example in the compilation of electronic circuits which had to reach predetermined outcomes [16]. It managed to even find diagrams of some patented solutions, which reached even better results than original once.

However our main problem was to build control logic of the experiment. As already mentioned in the introduction, the currently developed solutions works on the basis of the assembling of individual blocks of logic. Therefore one might consider a similar evolution as the compilation of the circuit from individual components. However, there is a problem, each block of control logic has its own strict rules of use. It can be only inserted into predetermined parts of control code, and they may/may not/must contain other specific blocks. This problem can be probably solved by an evolutionary algorithm code. However let's consider the obvious question, how to test the resulting outcomes? The only possible way would be to test the control logic by using the real experiment and the simulation of user's input to control the experiment. But what would happen if we get descendant with the bad qualities from the evolutionary algorithm? Consider the consequences of such descendant in the RE of water level control [17]. In this case the resulting file of control logic gives for example the ill signal when the liquid reaches the maximum level and the pump would not stop but rather increases its pumping speed. This behaviour of this kind of experiment could lead to malfunction or even to damage of the physical hardware (electrical short circuit because of the leakage of fluid). On this example we can see the evolutionary systems are not suitable for solving of our problem.

\section{B. Expert systems}

The name of the systems is in part derived from its intended function, which is to replace the function of an expert in a particular area. They provide advice, made decision or recommend a solution as a real expert in a real life would. To clarify uniqueness of this system we should first explain how would be this problem solved by the classical programming. The current computer programme processes the input data based on pre-defined decision algorithm and generates the results. The problem that arises here immediately is that the algorithms are adapted to the one kind of problem and allows only specific inputs in the exact format or the allowed range. Intelligence of the program itself rests directly in the source code, or their parts may be stored in a programme database.

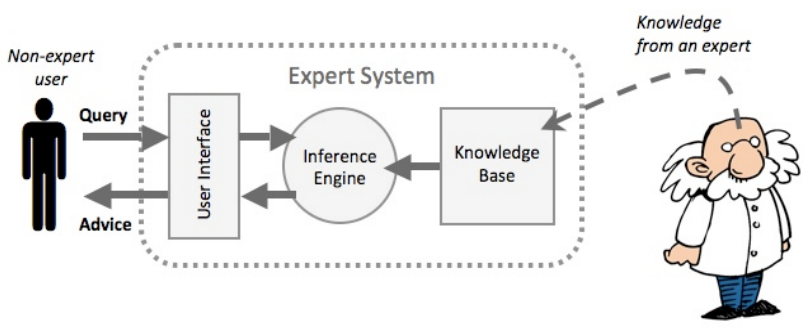

Figure 3. Basic scheme of ES [18]
The model of expert system (ES) is different (Figure 3). All intelligence (data, knowledge, information) are stored outside the source code in the "knowledge base". The source code is in charge with evaluating the situation on the basis of information from the knowledge base and inputs from the outside world. We can imagine the user's questions or information from sensors as inputs. The advantage of ES is that these inputs may not be precisely defined, and consequently ES may not offer clear answers (we can work with uncertain terms: rather not - don't know - rather yes). Another characteristic feature of expert systems is the explain ability (this ability the ES differs from its superset the "knowledge-based systems"). There are many types of expert systems, they may differ according to stored information in the knowledge base (rulesbased, not rules-based, hybrid) or according to the strategies of decision-making (forward or backward chaining).

Here are some concrete examples of ES usage in various sectors of human activity. We do not have to go far for example, in the company Skoda Plzen (author of the article comes from the Czech Republic) is used ES VIBROEXPERT to diagnose the vibration of steam turbine with high power ( $>50 \mathrm{MW})[19]$. In medicine, we can meet with a number of ES, for example the "Gideon" system which based on specific symptoms, determines the patients diagnosis. The system has been operational since 1995 and achieved 94\% success rate. [20] Another use of the health ES is "PEPID" which is able to design basic procedures for treating in more than 1100 cases of various health problems. It includes drug prescription, and their dosage [21].

Now let's consider the applicability of the ES in our problem of remote experiments. There is a wide range of uses both in the design and assembly of RE. Let's start from the design of the scheme of the experiment. It would be possible to build such ES so that on the basic requirements of the user the system proposes the arrangement of the appropriate RE. In this case, the user will probably by a teacher who wants to build an experiment for his students. It would be enough to say to the ES what phenomenon he wants to demonstrate or measure and which measuring modules he has. Such entry might look like this:

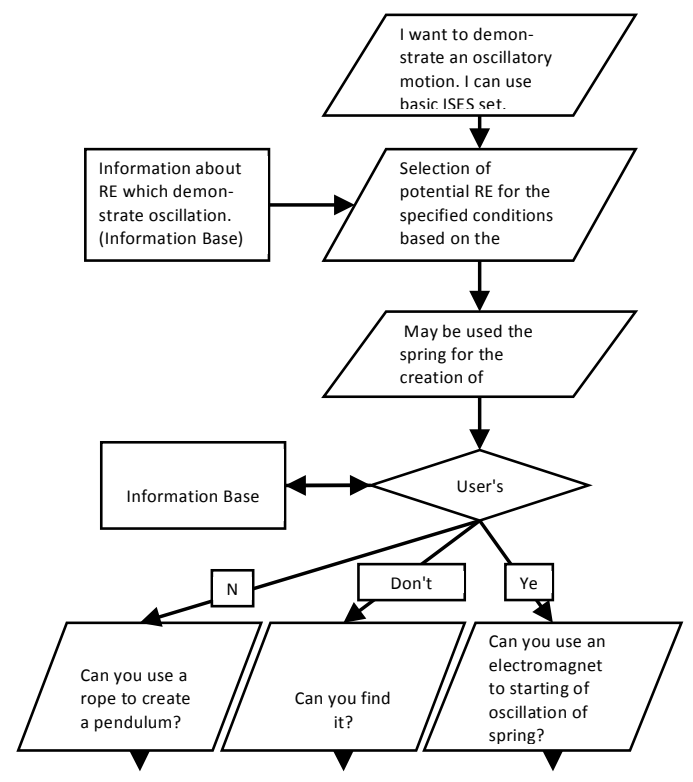

Figure 4. Scheme of possible procedure of Expert system 
In this way, the "interview" will be continued. It should be noted that this diagram was not pre-programmed and is created completely dynamically based on input parameters and a knowledge base of system. Note also that the ES works with vague expressions. The response of ES on "Don't know" answer of user could be the same as in diagram, or a completely different according to the importance of the query. From this phase of information obtaining, system could smoothly move to the assembly process of the experiment and lead the teacher in this activity.

Now let us approach and try to solve our problem. Depending on this "interview" ES would be able to assemble file with the control logic of the experiment. This could lead to immediate launch of RE with an already generated logic and user interface upon its completion.

This solution seems applicable to our problem. But how would the system correspond if we want to build an experiment absent in the knowledge base? In other words, if we want to design custom experiment. Can the system generate the control logic of RE in this case? If we included this option during the design of the system, it would perhaps propose some solutions. But the question remains to what extent the proposed solutions would include ideas of users which abound in their own creativity. To fulfil $100 \%$ of the user's ideas there would be necessary to transmit large amount of information. This would lead to already known solutions with the different ways of entering of information. Basically we can say that the use of ES would be appropriate in a remote experiment design if the work was carried out from the beginning to the end of design. That means from designing of linking the physical hardware to building of the RE's logic control. But only for the design of control logic, the EC don't simplify the work because the ideas and requirements of users can be completely arbitrary.

There arises the possibility of using an ES in a way that circumvents the problem of creating a file with control logic. We could replace the entire logic of remote experiment by ES. It would be very interesting and in some sense a unique solution. But for using in our case would be necessary to modify the whole control program of the ISES hardware to be controlled or replaced by ES. Also the developing of knowledge base seems difficult. Basic knowledge and information about the individual measuring modules and user input would be unified. But in the case of atypical experiment it was apparently necessary to add some set of information to a knowledge base that would complement description of experiment.

\section{Fuzzy logic}

Fuzzy logic is a subfield of the mathematical logic and is used in the artificial intelligence. In this logic, the logical statements are not limited to two values (true/false or $1 / 0)$ as in classical propositional logic. The statement is rated by the degree of truth, which may fall within the interval $<0,1>$. Let's take a simple example from everyday life: classification of water temperature. In the classical logic it is very difficult to determine the threshold at which the water changes from cold to hot water because this threshold is individual and depends on the person who evaluates it. For someone water may seem like hot at $30^{\circ} \mathrm{C}$ and for someone else may be this threshold shifted to $40^{\circ} \mathrm{C}$. Someone can also argue that water with a temperature of $20^{\circ} \mathrm{C}$ can be considered as cold. And exactly this is the case for which is the fuzzy logic intended. It is not fixed to the concept of classical propositional logic, but it allows classifying real-world situations. By using of fuzzy logic we obtain not only cold and hot states of water but also state such as tepid or warm. By this way we can accurately describe the actual state of water. For each statement, we can determine the degree of truth. Distribution of the statements for this example can be seen below in Figure 5.

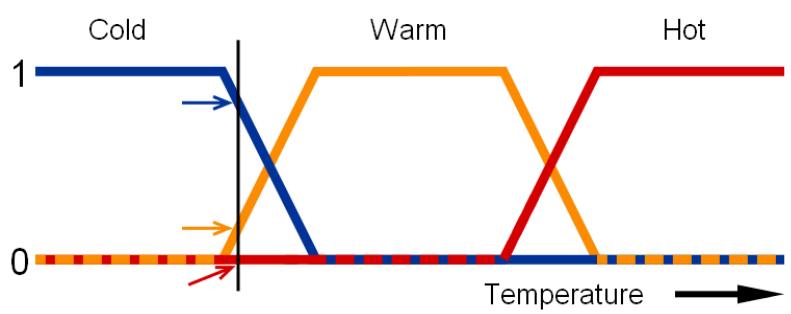

Figure 5. Distribution of the statements for water temperature

For this case we can classify the water by three statements with the degree of truthfulness in the point of the intersection of black line. The water is cold - $0.8(80 \%)$, the water is warm $-0.2(20 \%)$ and the water is hot -0 $(0 \%)$. So if we summarize the above description, we can say that the fuzzy logic allows working with vague data, and thus brings closer description of reality.

Fuzzy logic has an application in many fields. In the field of artificial intelligence, it has place mainly in the decision making and assessment of a certain situation. It is based on similar curves, as shown above. To each statement is assigned a membership function, which is usually represented by a particular geometric shape. It is trimmed to specific size based on the degree of truthfulness. By combining of geometric shapes of all statements we receive a new shape whose centre of gravity gives us rate of the resulting value $[22,23]$.

But it is possible to use the fuzzy logic in the making process of remote experiment? In this case, the authors' opinion is that it is not. On closer consideration of the principle of the experiment we find that there occur mainly unambiguous situations. For example, we cannot specify in any scale the value to which we should/ or should not connect a thermometer into the apparatus. We need the thermometer in the experiment or we don't, and any state between these two would not carry any information. It is similar with the control logic here. Perhaps we can use fuzzy logic to the selection of certain blocks of control code. But even here the decision takes the extreme values from the interval in most cases (yes I need this block / no I do not need it). Using of fuzzy logic here would be pointless. The last option of using of fuzzy logic is to replace the control logic of the experiment by fuzzy logic, which may bring positive results. Fuzzy logic is widely used in dealing with automatic controlling problems (fuzzyregulation) $[24,25]$. Therefore we can say that for automatic control type of experiments we can use it. But from my own experience I know that there are not many such experiments at a lower level of education. Therefore we can say that for our case is the fuzzy logic with very limited usefulness.

\section{One more step further- neural networks}

In the previous list of possibilities of artificial intelligence applications to RE was not mentioned one basic 
PAPER

method - neural networks (NN). To explain even basic principles of NN would be far from the scope of this paper, so we have to limit here to the description of general principles only and give the list of recommended literature with thorough basis of $\mathrm{NN}[26,27]$.

Neural networks are inspired by the human brain functioning. These networks, just like the brain consist of neurons, which are interconnected by ties. Signals are transmitted by using these links among neurons. Each neuron has a transfer function by which transforms the incoming signal to the output signal. Neuron can have a many inputs but only one output. Each input to a neuron has the assigned weight, which indicates the significance of the input to the output signal. The last parameter of the neuron, which is worth mentioning, is the threshold. This value indicates what size of the input signal is required to activate the neuron and allowing of passage to the output. The overall principle is "simple" to obtain the specific outputs for sets of inputs (learning sets) by adjusting the values of inputs weights. This process is referred to as learning of neural network. The advantage of neural networks is that after learning of particular type of task are able to correctly solve even those that were not included in the learning set $[26,27]$.

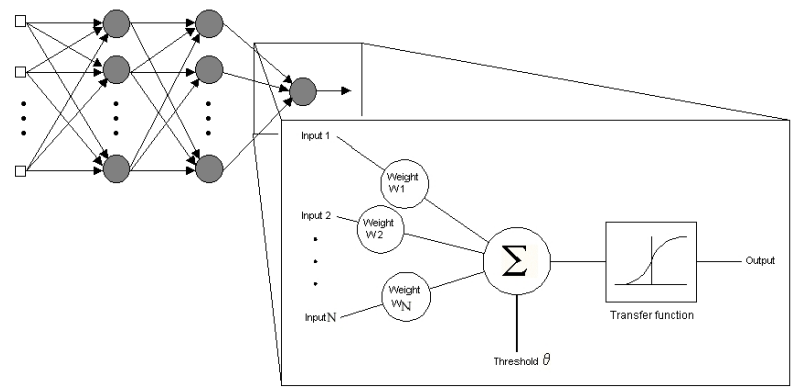

Figure 6. Scheme of a neural network (NN) with the detail of one neuron

The neural networks are used in a huge range of applications. Here are few of them: Typical application of neural networks is recognition of text and its conversion from graphical form to data form. Networks can be used for image compression, noise reduction or to predict the evolution of stock of exchange. We can also come across with the control of various robots, space vehicles, or even computer-controlled cars. A considerable part of the application of $\mathrm{NN}$ is also in the aerospace industry in many ways. Starts at forecasting occupancy of individual flights through the design of the aircraft structure and ends at the data evaluation and processing of various aircrafts parameters directly during the flight.

Let's look on the way of utilisation in the process of design of remote experiments, where at the first sight $\mathrm{NN}$ have enormous potential. Let us start from the design of RE. Here, the neural network could be used by the same way as an expert system, namely to offer the proper experiment, depending on the requirements of the teachers. NN can design the best experiment for your special situation, by gradually asking for the needed information.

If we want the NN to be a part of ISES hardware, it would be necessary to learn the NN designing of an experiment first. To achieve good results it would be necessary to involve a wide range of remote experiments in learning process of NN. The ensuing database would have to include the multitude of experiments connection schemes as well as their control logic. The problem is that for each hardware arrangement it is possible to find a large variety of types of the control logic. These types may vary in many details dependent on requirements of individual users. Complications also come with the possibility of connecting external, ad hoc necessary devices that are difficult to include. This also greatly increases the set of possible types of arrangements of logic. We can say that the creation of such a learning set would need prohibitively excessive efforts. Also it would require involving a multiple creative people to reach at least a majority of the categories of experiments that regular users may think of.

But, if this NN ran online, it could be programmed to learn more or less automatically, based on the requirements of users only. Then we get a very powerful tool for the design of remote experiments, which would be continuously updated by the users themselves. The network would be learned new experiments added by the users themselves in the evolvement of the system. In this way, the network could be enriched by the set of experiments' arrangements and their control logic formed by user, but also by the set of running experiments. In this way, it would be possible to allow the observation of the proposed experiment and the user would be able to verify whether the selected solution actually meets his/her requirements. For each newly inserted experiment a brief description would be added and also the key words that would represent a phenomenon, which is represented by the experiment. Such information would provide the additional parameter of the $\mathrm{NN}$ for the selection of appropriate experiment. After sufficient accumulation of information this NN would execute a complete design of RE based on the parameters set by the user.

This process may seem complicated, in particular with respect to the involvement of a sufficient number of users. But we have a unique opportunity in the situation, where many remote laboratories gather into one net, and create a sufficient learning set for NN automatically. So, we in principle can enrich the currently developed environment for creation of control logic by the auto-save function which saves arrangement of experiment even with the created control logic. All this information, together with a description of the experiment can be sent to our server where used to creation of a purpose oriented NN. This procedure will not simplify the building of the control logic of a RE at once, but in the long run it could remove the problem of the tedious control logic programming completely.

We cannot say that such a network as mentioned above would facilitate the creation of the control logic of the experiment. However, over time, the loading of control programs to the system would make their creation useless, because the users would derive the logic from existing once. Even if the proposed system has been operated worldwide, I dare to say that we would reach the point where the design of experiment absent in the database would be very rare. This approach would not be limited to the ISES hardware but could include the design of the RE for multiple physical hardware.

\section{E. And what about the future?}

The last chapter is devoted to a possible future in the field of RE and the trajectory it may take at present. Let us stick to the idea of a global system based on neural network, mentioned above. Such a project has a very large 
PAPER

application and the expansion options. Original design of the system with a rich library of experiments for teachers and students may be remade to the complex system creating nearly arbitrary REs. Measuring physical devices already possess even today the network connector by which they can be controlled across the Internet. If we had a set of such devices connected into one large system (the devices can be physically located all around the world), it would be possible to realize the actual setup of the experiment by the NN. The teacher or scientist would not need any own hardware but only connect to this system which would arrange everything for him and made the experiment available for use. The question is, whether this is the desired state indeed? We could in fact get to the point where even a teacher could not design such an experiment, not to mention his pupils. To what extent let this automation go? It seems that only time will tell.

Without discussion the future will extend the range of RE. Focus will move from the temperature and voltampere characteristic measurement, to controlling of complex robots and experiments representing the principles of quantum physics. Also, the creation of REs will extend far beyond educational institutions. This is already happening, for example the Google has recently published its project "Web Lab" [http://www.chromeweblab.com] which is nothing more than the laboratory of really exciting REs. . However the experiments created by such companies will reach a completely different scale than the experiments created at and for the primary schools. Since Google is a company that has extensive experience with applications of artificial intelligence in a wide range of their products, we can expect to find their application in this project also.

In the future, probably also encounter a voicecontrolled experiments. Voice control has made a great progress for the last decade. A large share of this progress has Google Company, who applied speech recognition in their mobile operating system Android. From my own experience I can say that it achieves very good recognition results. So I see no reason why this trend should not continue and extend into the REs.

Far future will then likely change the overall view to the RE. We will no longer operate the devices via a web interface and watch it with the web camera. We will be pulled directly into the virtual science lab, by using a virtual reality, where we will work with devices and experiments as we did in the real world. We will be able to get even further, for example, we will be able to zoom at the heating of the substance to see the movements of its individual atoms. Or follow the course of particle collisions in collider. But all this is a far in future. Or not so far away?

\section{CONCLUSIONS}

The paper brought a number of possible improvements and modifications of the emerging system for the creation of control logic for remote experiment based on ISES system. At this point, we are to some extent limited by control software whose redesign would take very much time. Therefore it is necessary to stay at this concept which for its operation requires a file with control logic. For this reason, we can exclude the options which included the replacement of the proceedings by some of AI methods mentioned in the article.
Automatic compilation of control logic by any of the above mentioned methods could be probably realized. It would not be possible to implement creation of logic for any types of experiments. These can greatly vary depending on the creativity of teachers. Therefore, the coverage of all possible combinations is very complicated. This problem could be resolved by neural network. However, it would require a large learning set. Unfortunately it is currently not available.

So there is a last resort, namely the realization of the whole design of the experiment with its choosing from some library. Because there is no library of experiments with which we could work at this moment, the procedure will be as follows. To the formed development environment will be added the feature that will automatically record the connected measuring devices and also save all the control logic that will be created for this arrangement. Because the user is asked to enter a name and a brief description of the experiment, there is no problem to record these information which leads to better classification of the collected data. Such collected data will be sent to the server where they will be collected. In this version of software there will be the opportunity to join this online library and be inspired by one of the stored arrangement and prepared logic.

By this way, there will be obtained a large data set that will be used in future versions of the development environment. Either as an information base for expert system or as a database from which will be selected experiment, that will best meet the requirements of the teacher, by using of selected methods (such as neural networks). Further development of this system will be based on popularity among users and the number of their contributions to the online library of experiments.

\section{REFERENCES}

[1] Humos, A. A., Alhalabi, B., Hamzal, M., Shufro, E., \& Awada, W.(2005). Remote labs environments (RLE): A constructivist online experimentation in science, engineering, and information technology. In Proceedings of IECON 2005. 32nd Annual Conference of IEEE, Industrial Electronics Society (pp. 2156-2161). Raleigh, NC: IEEE

[2] C. Gravier, J. Fayolle, B. Bayard, M. Ates, and J. Lardon, State of the art about remote laboratories paradigms - foundations of ongoing mutations, International Journal of Online Engineering Vol. 4, No. 1 (2008) 19-25, http://online-journals.org/i-joe/article/view/ $\underline{480}$

[3] Auer M.E. and Gravier C. (2009 October-Decmber), The Many Facets of Remote Laboratories in Online Engineering Education IEEE Trans. Learn. Techn. Vol. 2, No. 4, p.260

[4] Schauer, F., Lustig, F., Dvorák, J., Ožvoldová, M.: Easy to Build Remote Laboratory with Data Transfer using ISES - Internet School Experimental System, Eur. J. Phys. 29 (2008) 753-765, ISBN 978-0-9741252-9-9]

[5] František Schauer, František Lustig, Miroslava Ožvoldová: Internet Natural Science Remote e-Laboratory (INRe-L) for Remote Experiments /.In: Innovations 2011: World Innovations in Engineering Education and Research (USA), iNEER / ed. W. Aung, et al. - (2011), s.51-68.,]

[6] Krbecek, Michal. Creation of multimedia interactive teaching tool with utilisation of remote experiments. Zlín, 2011. 108 s. Diploma thesis. TOMAS BATA UNIVERSITY IN ZLÍN.

[7] KRBEČEK, Michal, František SCHAUER a Roman JAŠEK. Security Aspects of Remote e-Laboratories. International Journal of Online Engineering (iJOE). 2013-07-11, vol. 9, issue 3, s. 3439. DOI: 10.3991/ijoe.v9i3.2586. Available from: http://onlinejournals.org/i-joe/article/view/2586 
PAPER

Possible Utilization of the ARTIFICIAL INTELLIGENCE ELEMENTS IN THE CREATION OF REMOtE EXPERIMENTS

[8] Cooper M 2005 Remote laboratories in teaching and learning issues impinging on widespread adoption in science and engineering education, iJOE Intern., J. Onl. Egin. 11.

[9] Library of Labs. LiLa - Library of Labs [online]. Dec 13, 2011 [Read: 2013-08-12]. Accessible from: http://www.lilaproject.org/home.html

[10] F. Schauer, I. Kurritka and F. Lustig, "Creative Laboratory Experiments for Basic Physics Using Computer Data Collection and Evaluation Exemplified on the Intelligent School Experimental System (ISES)," in Innovations 2006, World Innovations in Engineering Education and Research, iNEER Special Volume, W. Aung et al. (eds.), pp. 305-312, USA, 2006

[11] National Instruments. National Instruments [online]. 2012 [Read: 2013-08-12]. Accessible from: http://www.ni.com/labview/

[12] Agilent Technologies. Agilent Technologies [online]. 2000-2012 [Read: 2013-08-12]. Accessible from: http://www.home. agilent.com/

[13] M. Krbecek, F. Schauer and F. Lustig, EASY REMOTE ISES Environment for Remote Experiments Programming, Innovations 2013: World Innovations in Engineering Education and Research, W. Aung, et al. (eds.), iNEER, Arlington, VA, 2013, in press

[14] Charles Darwin. Charles Darwin: Darwin online [online]. 2002 [Read: 2013-08-12]. Accessible from: http://darwin-online.org.uk/

[15] Zelinka, Ivan. Umělá inteligence v problémech globální optimalizace. 1. vyd. Praha: BEN - technická literatura, 2002, 189 s. ISBN 80-730-0069-5.

[16] Xuesong Yan, Qinghua $\mathrm{Wu}$-, Chenyu $\mathrm{Hu}$ - a Qingzhong Liang -. Design Electronic Circuits Using Evolutionary Algorithms. Journal of Next Generation Information Technology. 2010-5-31, Vol. 1, n. 1, p. 127-139. ISSN 2092-8637. DOI: 10.4156/ jnit.vol1.issue1.11. Accessible from: http://www.aicit.org/jnit/ paper_detail.html?q=14

[17] Water level control Experiment. ISES [online]. 2012 [Read: 201308-12]. Accessible from: http://www.ises.info/index.php/en/ laboratory/experiment/water-level-control, UTB in Zlin.

[18] What is an Expert System. What is an Expert System [online]. 2012 [Read: 2013-08-12]. Accessible from: http://www.igcseict.info/theory/7_2/expert/index.html
[19] The utilisation of expert systems in the diagnosis of machinery [online]. $2010 \quad$ [Read: 2013-08-12].Accessible from:http:/www.cmms.cz/vibrani-diagnostika/181-vyuzitiexpertnich-systemu-v-diagnostice-strojnich-zarizeni.html

[20] GIDEON. GIDEON: Helping doctors make better decisions [online]. 1994 - 2012 [Read: 2013-08-12]. Accessible from: http://www.gideononline.com/

[21] PEPID. PEPID: medical information resources [online]. 2012 [Read: 2013-08-12]. Accessible from: http://www.pepid.com/

[22] DANIEL MCNEILL, Paul Freiberger. Fuzzy logic. 1st Touchstone ed. New York: Simon, 1994. ISBN 06-718-7535-3.

[23] ROSS, Timothy J. Fuzzy logic with engineering applications. New York: McGraw-Hill, 1995. ISBN 00-705-3917-0.

[24] Research on Fuzzy Regulation Strategies in the Constant Air Volume Air Conditioning System. Control Systems for Energy Efficiency and Comfort [online]. 2006, V-1-2 [Read: 2013-08-12]. Accessible from: http://hdl.handle.net/1969.1/5288

[25] Fuzzy Regulation for the Intelligent Control of Switching-Mode Buck Power-Electronic Converter Using Genetic Algorithm-Based Tuning. IMECS 2012 [online]. 2012, II [Read: 2013-08-12]. DOI: $9789881925190 . \quad$ Accessible from: http://www.iaeng.org/publication/IMECS2012/IMECS2012 pp83 9-859.pdf

[26] ANDERSON, James A. Introduction to neural networks. 3. printing. Cambridge, Mass: Bradford Books, 1995. ISBN 02-625-10812 .

[27] GURNEY, Kevin. An introduction to neural networks. Boca Raton: CRC Press, 1997, xi, 234 s. ISBN 978-1-85728-503-1.

\section{AUTHORS}

M. Krbeček, F. Schauer and I. Zelinka are with the Tomas Bata University in Zlín, Faculty of Applied Informatics, Nad Stráněmi 4511, Zlín, CZ- 760 05, Czech Republic (e-mail: krbecek@fai.utb.cz, fschauer@fai.utb.cz, zelinka@fai.utb.cz).

Submitted, 12 August 2013. Published as re-submitted by the authors 23 January 2014. 